\title{
Lasers and Electro-optics for Ground-based Astronomy
}

\author{
Richard G. Dekany \\ Caltech Optical Observatories, California Institute of Technology, \\ 1200 East California Boulevard, Pasadena, CA 91125, USA \\ rgd@astro.caltech.edu
}

\begin{abstract}
To expand their science reach, terrestrial observatories have long demanded innovative optical technologies. Today, photonics enabling the active control, compensation, and detection of astronomical light are once again opening new vistas on our Universe.
\end{abstract}

\section{Introduction}

Modern astronomy has benefited from successive waves of technical innovation. Beginning with the advent of large optics fabrication, and followed by successive periods of rapid advancement in detector technology for visible, then infrared, and now extending to other wavebands, each technology wave has multiplied the science reach of terrestrial astronomical observatories. Most recently, atmosphere-compensating adaptive optics technologies exploit a range of photonics technologies, including highpower lasers, optical modulators, and fiber calibration systems.

\section{Laser Guide Star Adaptive Optics}

Astronomical light reaching the Earth, sometimes having traveled for billions of years and reaching an exquisite level of wavefront phase flatness, impacts upon the Earth's atmosphere and in grotesquely aberration during its final millisecond of travel before reaching terrestrial telescopes, spoiling image quality which in turn compromises spatial resolution and image contrast for astronomical science.

\section{Lasers}

Adaptive optics (AO), the sensing and active shaping of the optical phase and occasionally the amplitude of an optical wave (e.g. the wavefront), is now a mainstream capability at all 8-10 meter diameter optical/infrared telescope observatories and is considered essential to the coming generation of 25-40 meter telescopes [1,2]. Refereed astronomical science publications enabled by AO systems now number in the hundreds per year [3]. Still, the availability of appropriately bright sources, needed to provide accurate and rapid measurement of the Earth's turbulent atmosphere, is highly limited. High-powered lasers are now used to expand the fraction of sky available to AO correction, with the frontiers of development encompassing increased laser 


\section{PWB2.pdf}

power, field ruggedization, and lower lifecycle costs. As ever increasing sensitivity and observing contrast demands drive the technical requirement of improving wavefront flatness parameterized by $\sigma$, the time-averaged RMS wavefront error over the telescope pupil, the correspondingly required laser power scales to first order as [4]

$$
\text { Laser power } \propto \sigma^{-\frac{18}{5}} \text {. }
$$

Thus, equivalent compensation at blue (e.g. $400 \mathrm{~nm}$ ) wavelengths requires hundreds of times the laser power in use to compensate near-infrared (e.g. $1600-2400 \mathrm{~nm}$ ) wavelengths today, dauntingly ultimately requiring kilowatts of average power. Furthermore, the largest telescopes in the world require resonant excitation of the mesospheric layer of sodium atoms, requiring specialized wavelength, frequency, and pulse formats.

\section{Deformable Mirrors and Phase Modulators}

In order to provide broad wavelength compensation to astronomical light, astronomical AO correction has traditionally been applied using deformable facesheet and membrane mirrors (DM's). Recent advances in microelectromechanics systems (MEMS) manufacturing of DM's has opened a new design space for instrumentation. Improvements in spatial light modulator (SLM) technology, including speed, optical transmission, and thermal control, could result in increased application in astronomy, including uplink or broadcast AO laser compensation.

\section{References}

1. Dekany, R., Britton, M., Ellerbroek, E., Gavel, D., Herriot, G., Max., C., Nelson, J., and Veran, J.-P., "Adaptive Optics Requirements Definition for TMT," Proc. SPIE 5490 (2004).

2. Ellerbroek, B., et al., "A conceptual design for the Thirty Meter Telescope adaptive optics systems”, Proce. SPIE $\underline{6272}$, (2006).

3. Dekany, R. and Lloyd-Hart, M., Eds., A Roadmap for the Development of United States Astronomical Adaptive Optics, 2008, available at: http://www.aura-astronomy.org/nv/AO_Roadmap2008_Final.pdf

4. Dekany, R. G., Bauman, B. J., Gavel, D. T., Troy, M., Macintosh, B. A., Britton, M. C., "Initial concepts for CELT adaptive optics," Proc. SPIE $\underline{4839}$, p. 1165 (2003). 